\title{
Non-dystrophic myotonias: clinical and mutation spectrum of 70 German patients
}

\author{
Noemi Vereb $^{1} \cdot$ Federica Montagnese ${ }^{1} \cdot$ Dieter Gläser $^{2} \cdot$ Benedikt Schoser $^{1} \mathbb{C}$
}

Received: 25 June 2020 / Revised: 9 November 2020 / Accepted: 19 November 2020 / Published online: 2 December 2020

(c) The Author(s) 2020

\begin{abstract}
Introduction Non-dystrophic myotonias (NDM) are heterogeneous diseases caused by mutations in CLCNI and SCN4A. The study aimed to describe the clinical and genetic spectrum of NDM in a large German cohort.

Methods We retrospectively identified all patients with genetically confirmed NDM diagnosed in our center. The following data were analyzed: demographics, family history, muscular features, cardiac involvement, CK, EMG, genotype, other tested genes, treatment perceived efficacy.

Results 70 patients (age 40.2 years $\pm 14.9 ; 52.8 \%$ males) were included in our study (48 NDM-CLCN1, 22 NDM-SCN4A). The most frequent presenting symptoms were myotonia (NDM-CLCN1 83.3\%, NDM-SCN4A 72.2\%) and myalgia (NDMCLCN1 57.4\%, NDM-SCN4A 52.6\%). Besides a more prominent facial involvement in NDM-SCN4A and cold-sensitivity in NDM-CLCN1, no other significant differences were observed between groups. Cardiac arrhythmia or conduction defects were documented in sixNDM-CLCN1 patients (three of them requiring a pacemaker) and one patient with NDM-SCN4A. CK was normal in $40 \%$ of patients. Myotonic runs in EMG were detected in $89.1 \%$ of CLCN1 and $78.9 \%$ of SCN4A. 50\% of NDM-CLCN1 patients had the classic c.2680C >T (p.Arg894*) mutation. 12 new genetic variants are reported. About $50 \%$ of patients were not taking any anti-myotonic drug at the last follow-up. The anti-myotonic drugs with the best patient's perceived efficacy were mexiletine and lamotrigine.

Conclusion This study highlights the relevant clinical overlap between NDM-CLCN1 and NDM-SCN4A patients and warrants the use of early and broad genetic investigation for the precise identification of the NDM subtype. Besides the clinical and genetic heterogeneity, the limited response to current anti-myotonic drugs constitutes a continuing challenge.
\end{abstract}

Keywords Non-dystrophic myotonia $\cdot$ Channelopathies $\cdot$ Myotonia congenita $\cdot$ CLCN1 $\cdot S C N 4 A$

\section{Introduction}

Non-dystrophic myotonias (NDM) are rare hereditary neuromuscular diseases caused predominantly by mutations in $C L C N 1$ or SCN4A, respectively coding for the voltage-gated

Noemi Vereb and Federica Montagnese contributed equally.

Supplementary Information The online version contains supplementary material available at https://doi.org/10.1007/s0041 5-020-10328-1.

Benedikt Schoser

Benedikt.Schoser@med.uni-muenchen.de

1 Friedrich-Baur-Institute, Department of Neurology, LudwigMaximilians-University, Ziemssenstrasse 1a, 80336 Munich, Germany

2 Genetikum, Neu-Ulm, Germany muscle channels $\mathrm{ClC}-1$ and $\mathrm{NaV} 1.4$ [1, 2]. More than 150 CLCN1 and at least 100 SCN4A myotonia-associated gene variants are currently known [3]. The common feature of these diseases is the altered electrical excitability of the muscular membrane [4]. In most cases, mutations lead to hyperexcitability, which manifests itself clinically as delayed muscle relaxation after voluntary contraction, also called myotonia [5-7]. The pathophysiological mechanism of myotonia is mainly related to reduced activity of the chloride channels and consequently reduced chloride conductivity in CLCN1, whereas in SCN4A-myotonias it is caused by an impaired inactivation of the NaV1.4 channels $[5,8]$.

CLCN1-myotonias, also known as myotonia congenita (MC), are to be distinguished into the autosomal dominant type Thomsen (TMC) and the autosomal recessive type Becker (BMC), related to the type of mutation. Although some CLCN1 mutations may be inherited both as autosomal 
recessive and dominant, making it difficult in some patients to classify them without a detailed genetic and clinical study of other family members. SCN4A-myotonias include paramyotonia congenita Eulenburg (PMC), potassium aggravated myotonia (PAM), and hyperkalemic periodic paralysis with myotonia (hyperPP) [9].

The phenotype of NDMs involves almost exclusively the skeletal muscle and, differently from dystrophic myotonias (DM), no relevant extra-muscular involvement has been described so far. The onset of symptoms is usually described in the first to the second decade of life; the lead symptom is myotonia, which is however often described by patients as stiffness, occurring especially during suddenly initiated movements after a resting period [9]. Otherwise, prolonged depolarization may temporarily reduce the excitability of the muscle membrane, causing transient weakness or periodic paralysis [8]. Significant clinical overlap exists between CLCN1 and SCN4A myotonias. Nevertheless, some features can help to distinguish both forms. CLCN1-myotonias are usually characterized by a warm-up phenomenon, worsening of myotonia under low temperature environment, and more prominent myotonia at lower limbs. Temporary weakness and segmental muscle hypertrophy occur mainly in patients with BMC [2, 10-12]. On the other hand, patients with PMC typically show worsening of their myotonia after repetitive movements, and under low-temperature environment. The SCN4A associated myotonia commonly involves the facial muscles with eyelid myotonia; the muscle weakness can occur more frequently and may persist longer than in CLCN1 patients $[2,11]$. In addition to medical history and neurological examination, the detection of myotonic runs (MR) in electromyography (EMG) is an important hallmark for suspecting a NDM. Specific EMG protocols (repetitive stimulation, the "short" and "long" exercise tests, and the provocative cold test) even allow the differentiation between chloride and sodium channel myotonia with relatively high sensitivity and specificity [13]. Nevertheless, the precise diagnosis needs genetic analysis.

Up-to-date, no causal treatments are available for NDM but several drugs can be adopted for the symptomatic treatment of myotonia in the attempt to improve patients' quality of life [2]. Presently, anti-myotonic drugs with the highest level of evidence concerning efficacy are mexiletine and lamotrigine [14-16]. Many other substances such as carbamazepine, flecainide, phenytoin, propafenone, or more recently cannabinoids are also being used [11, 17]. Unfortunately, many patients still do not report sufficient improvement of their core symptom myotonia.

Due to the rarity of these diseases, only a few large cohorts $(n>50)$ have been described in the literature to this date (Table 1). A description of a large genetically confirmed cohort of German NDM patients dates back to the year 1993 [18].
The aim of this study was to investigate the clinical features of a large cohort of German patients with NDM, highlighting some diagnostic and therapeutic challenges and expanding the genotype by describing newly detected genetic variants.

\section{Patients and methods}

\section{Patients}

This is a monocentric, retrospective study, performed at the Friedrich-Baur-Institute, Munich, Germany. We have included patients of all ages that, according to our institutional database of patient records, had a genetically confirmed diagnosis of NDM. If new genetic variants were detected, their pathogenicity was evaluated considering the presence of positive family history, documented typical clinical signs or symptoms of NDM, and the presence of myotonic runs in EMG. The database search refers to data archived between the years 1994 to 2019. Additional clinical data were obtained by additionally reviewing paper records of the identified patients.

\section{Methods}

The following data were collected and analyzed: demographics, age at onset, family history, first symptom, symptoms present during disease progression (myotonia, weakness, muscle hypertrophy and atrophy, myalgia, cramps, dysphagia, dysphonia), symptoms at the last follow-up visit, presence of any type of cardiac involvement, creatine kinase (CK), EMG, genetic results including previously tested genes, diagnostic delay (time between the onset of symptoms and the genetic diagnosis), past and current anti-myotonic medications with patients perceived efficacy.

For disease onset, either the exact year or the development stage (e.g., birth, infancy, childhood...) were collected according to the information available from patient' records. The age groups were determined as follows: $<1$ year as infant, 1-9 years as child, 10-17 years as adolescent, $18-35$ years as young adult, $36-55$ years as middle-aged adult, and $>55$ years as old adult $[19,20]$. We combined family history, clinical features, EMG, and genetic results to classify patients with CLCN1 mutations in either Becker (BMC) or Thomsen myotonia (TMC).

The following cardiac abnormalities were included: conduction defects, cardiac arrhythmia, or cardiomyopathies.

Due to various reference values of CK through the years, all values were expressed as the percentage of normal value and converted into absolute values considering gender-specific reference values of our laboratory. The CK is reported referring to the highest $\mathrm{CK}$ values found. The EMG data 


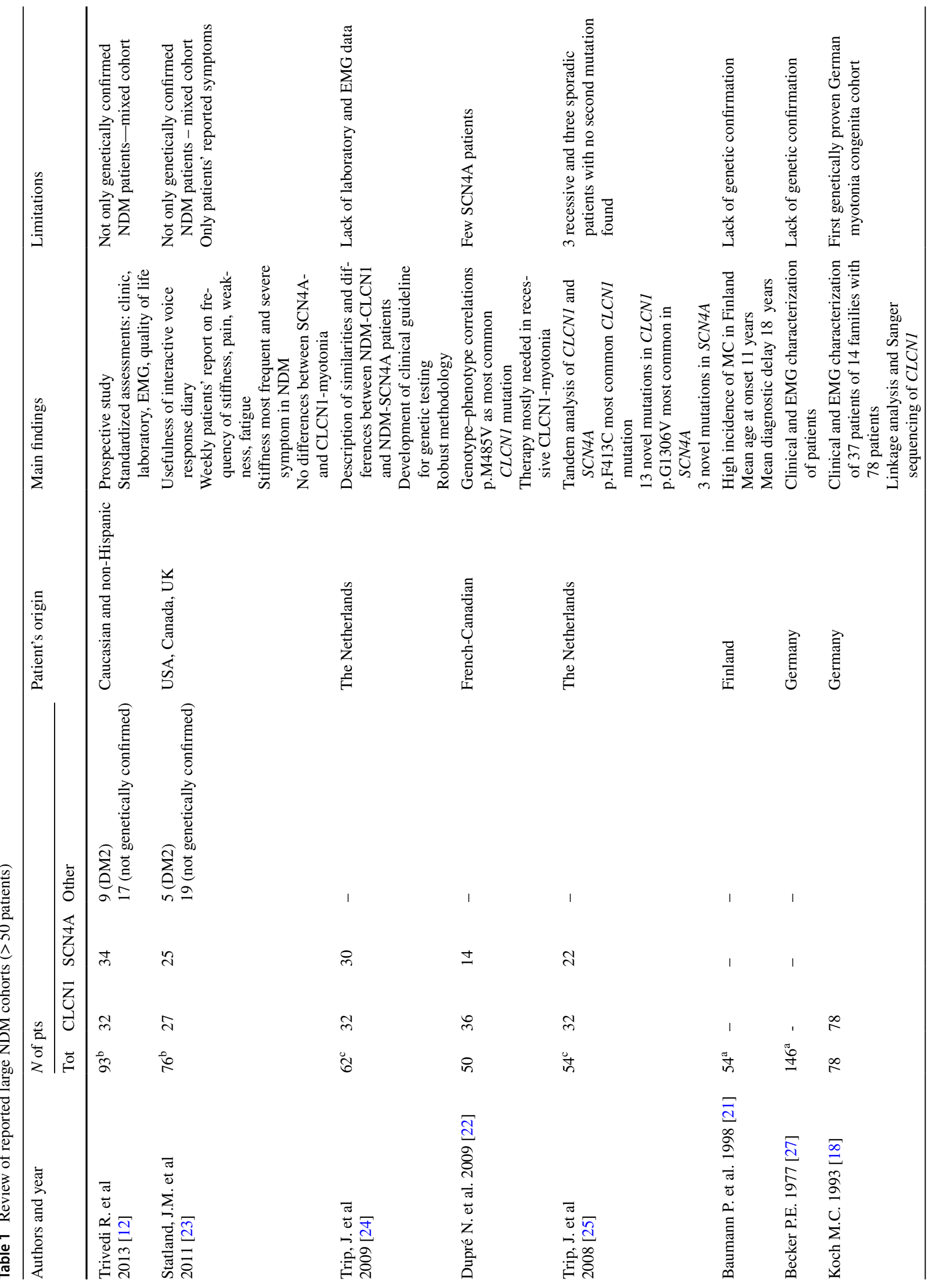




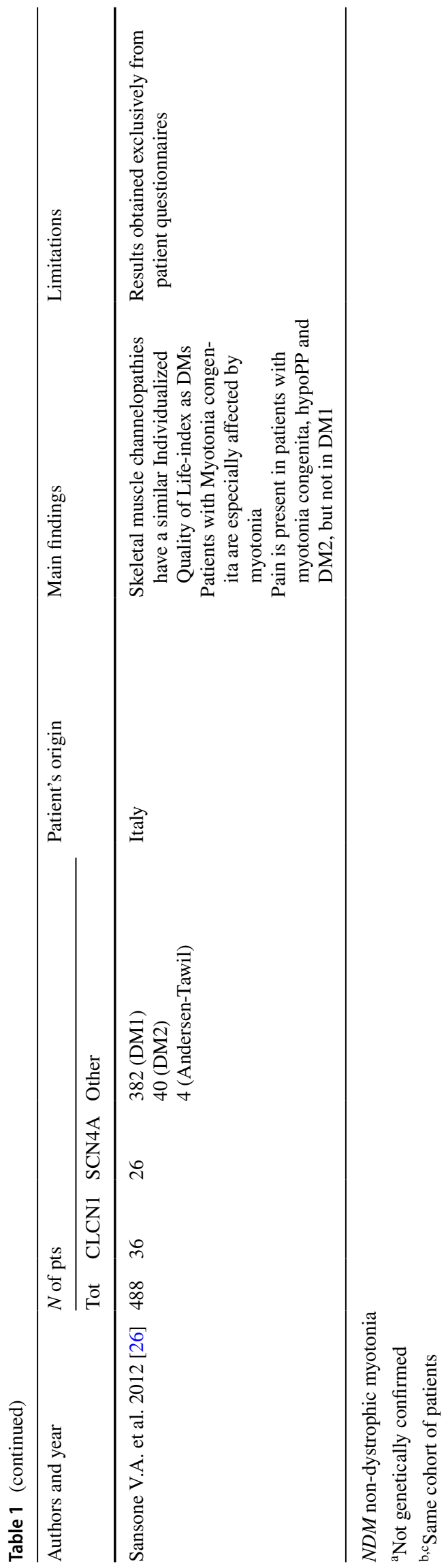

refer to the first documented EMG. The efficacy of antimyotonic treatment was semiquantitatively evaluated as $1=$ excellent, $2=$ good, or $3=$ poor according to the patient evaluation reported in patient records by the treating doctor. The follow-up period was calculated between the first and the last recorded follow-up visit.

\section{Statistic analysis}

All statistical analyses were performed with IBM SPSS Statistics (Version 25.0.0.1). The normality of variables was assessed by Shapiro-Wilk-test. Descriptive analysis included mean \pm standard deviation (SD) or median and interquartile range (IQR), as appropriate, for continuous variables. Frequencies were calculated for categorical variables. For normally distributed continuous variables unpaired Student's tests were used to assess differences between groups, while for non-normally distributed continuous variables the Mann-Whitney $U$ test was used. $\chi^{2}$ or Fisher's exact test was used to compare frequencies. All hypothesis tests conducted were two-tailed. A $p$ value $<0.05$ was considered significant.

\section{Results}

\section{Clinical features}

We identified 76 patients with clinical suspicion of NDM and genetic results. Six patients were excluded due to inconclusive genetic results or lack of sufficient clinical data. We enrolled 70 patients (64 families): $48(68.5 \%)$ patients had a diagnosis of CLCN1-myotonia and 22 (31.4\%) of SCN4Amyotonia. The mean age was $40.2 \pm 14.9$ years, with no significant difference between the two groups. Thirty-seven patients were male (52.8\%). No gender differences were identified for any of the analyzed variables. A detailed summary of the core clinical features of this cohort and the differences between NDM-CLCN1 and NDM-SCN4A are shown in Table 2 and Fig. 1.

The onset of symptoms was reported mostly in childhood (39.6\%) or adolescence $(28.5 \%)$ and no patient reported the onset of symptoms after the fifth decade. No statistically significant difference was observed in age at onset between NDM-CLCN1 and NDM-SCN4A. The most frequent symptom at onset was myotonia $(74.1 \%)$, followed by myalgia (16.1\%) and muscle weakness (14.5\%). Myotonia at onset was more frequent in patients with CLCN1-myotonia $(85.7 \%)$ than in patients with SCN4A-myotonia $(50 \%)$ $(p=0.003)$; periodic paralysis was only reported in patients with SCN4A-myotonia (20\%) $(p=0.008)$.

At the last documented follow-up visit, the majority of patients presented myotonia (NDM-CLCN1 83.3\%, NDMSCN4A $72.2 \%, p=0.31$ ) and up to $25 \%$ of patients presented 
Table 2 Demographics and clinical features of this cohort of patients

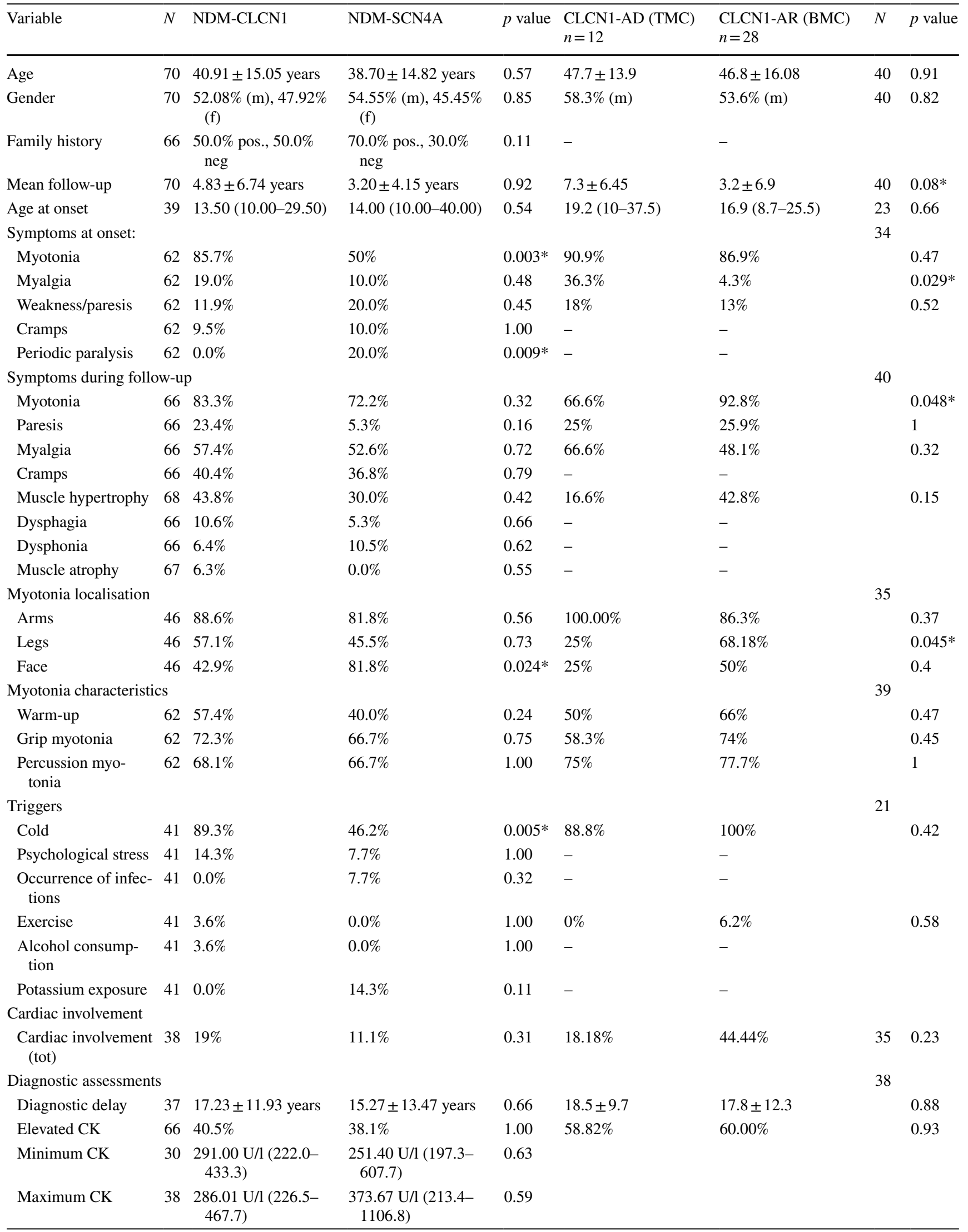


Table 2 (continued)

\begin{tabular}{|c|c|c|c|c|c|c|c|c|}
\hline Variable & $N$ & NDM-CLCN1 & NDM-SCN4A & $p$ value & $\begin{array}{l}\text { CLCN1-AD (TMC) } \\
n=12\end{array}$ & $\begin{array}{l}\text { CLCN1-AR (BMC) } \\
n=28\end{array}$ & $N$ & $p$ value \\
\hline EMG & & & & & & & 38 & \\
\hline Myotonic runs & 65 & $89.1 \%$ & $78.9 \%$ & 0.43 & $90.9 \%$ & $100 \%$ & & 0.29 \\
\hline $\begin{array}{l}\text { Complex-repetitive } \\
\text { discharges }\end{array}$ & 65 & $4.3 \%$ & $10.5 \%$ & 0.57 & & & & \\
\hline $\begin{array}{l}\text { Other pathologi- } \\
\text { cal spontaneous } \\
\text { activity }\end{array}$ & 65 & $13.0 \%$ & $15.8 \%$ & 0.71 & & & & \\
\hline
\end{tabular}

*Statistically significant

Fig. 1 Comparison of symptoms in patients with NDMCLCN1 and NDM-SCN4A. *Statistically significant; filled: CLCN1; Dotted: SCN4A-(created with Excel)

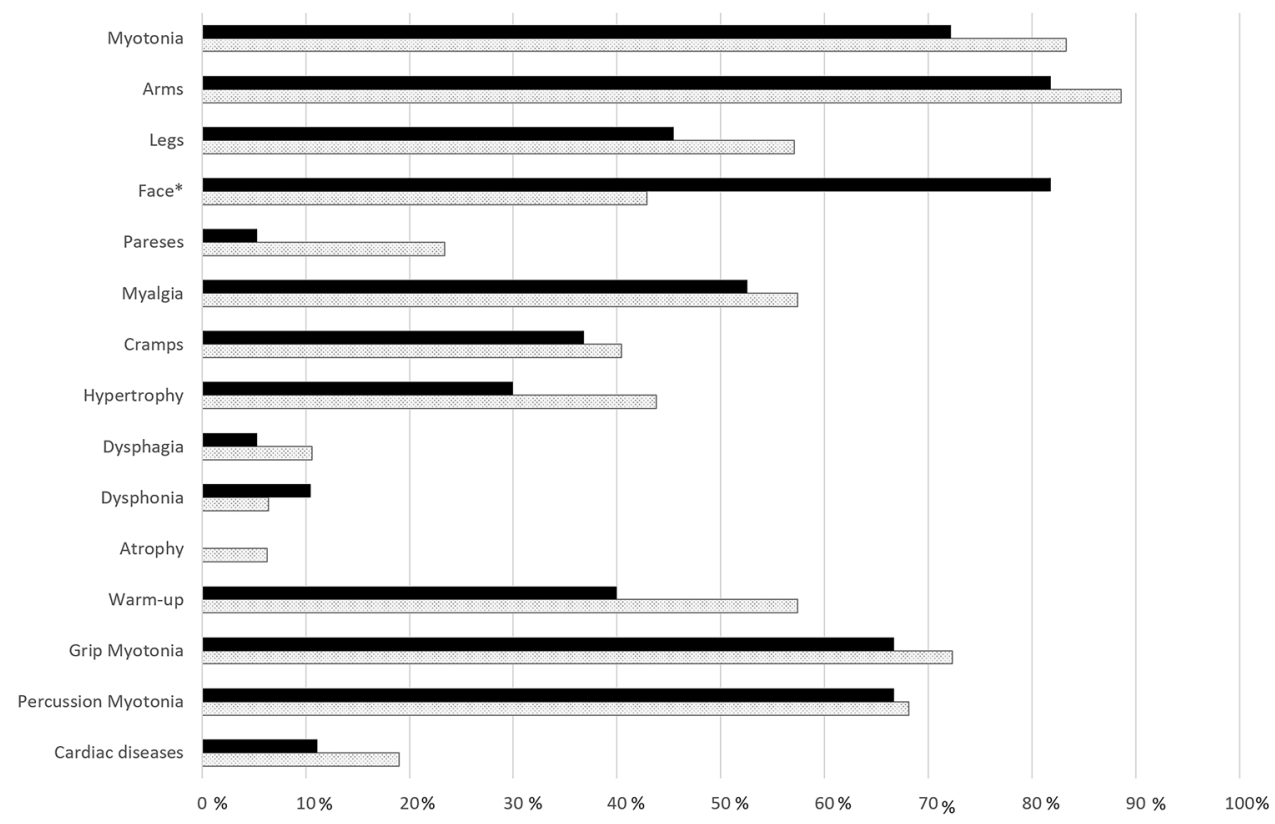

muscle weakness at their final neurological examination (NDM-CLCN1 23.4\%, NDM-SCN4A 5.2\%, $p=0.15$ ). We could not find any statistically significant changes of manual muscle testing between the first and last examinations $(p=0.184)$. The distribution of myotonia is reported in Table 2, facial involvement was significantly more frequent in patients with SCN4A-myotonia (81.8\% vs. $42.8 \%$, $p=0.024)$. Cold temperature environment was the most common trigger for myotonia in all patients $(75.61 \%)$, being however more frequently reported as a factor triggering or worsening symptoms from NDM-CLCN1 patients $(89.2 \%$ vs. $46.1 \%, p=0.005)$.

More than half of the patients reported myalgia during the disease course (NDM-CLCN1 57.4\%, NDM-SCN4A $52.6 \%, p=0.72$ ). Additionally, about $30-40 \%$ of patients reported muscle cramps with no significant differences between both types. Dysphagia and dysphonia were only rarely reported and mostly mild. Muscle hypertrophy was documented in both subtypes (NDM-CLCN1 $43.7 \%$, NDM-SCN4A 30\%, $p=0.41$ ), whereas muscle atrophy was only documented in a few NDM-CLCN1-patients $(6.2 \%)$.

In the NDM-CLCN1 cohort, we classified 28 patients as BMC, 12 patients as TMC and in 8 cases it was not possible to clearly classify them as BMC or TMC. Comparing the features of BMC and TMC we found significant differences only as regards the presence of myalgia (more common in TMC), the presence of myotonia (more prevalent in $\mathrm{BMC}$ ), and the distribution of myotonia, more frequently occurring in the legs of BMC patients (Table 2). Cardiac involvement was reported in six patients with NDMCLCN1 and one patient with NDM-SCN4A (Table 2). A detailed description of the cardiac pathologies is reported in Table 3. Six NDM-CLCN1 patients had relevant cardiac arrhythmias, in one with an early onset before age 45 . Of these, three required pacemaker implantation. 
Table 3 Cardiac involvement

\begin{tabular}{|c|c|c|c|c|c|c|c|}
\hline Pt. ID & Gender & Age & NDM & $\begin{array}{l}\text { Onset of car- } \\
\text { diac involve- } \\
\text { ment }\end{array}$ & Cardiac involvement & Mutation 1 & Mutation 2 \\
\hline 8 & M & 56 & CLCN1 & 54 & $\begin{array}{l}\text { Complete right bundle branch } \\
\text { block }\end{array}$ & c. $2680 \mathrm{C}>\mathrm{T}($ p.Arg894*) & - \\
\hline 10 & $\mathrm{~F}$ & 48 & CLCN1 & 45 & Cardiac arrhythmia & c. $870 \mathrm{C}>\mathrm{G}$ (p.Ile290Met) & - \\
\hline 17 & M & 68 & SCN4A & Not known & Mild cardiac arrhythmia & c. $1333 \mathrm{G}>\mathrm{A}$ (p.Val445Met) & - \\
\hline 29 & $\mathbf{F}$ & 78 & CLCN1 & Not known & $\begin{array}{l}\text { Global heart failure and pace- } \\
\text { maker implantation unknown } \\
\text { cause }\end{array}$ & c. $2680 \mathrm{C}>\mathrm{T}\left(\mathrm{p} . \operatorname{Arg} 894^{*}\right)$ & c. $1238 \mathrm{~T}>\mathrm{G}$ (p.Phe413Cys) \\
\hline 32 & M & 56 & CLCN1 & Not known & $\begin{array}{l}\text { Cardiac arrhythmia, } \mathrm{AV} \text {-block } \mathrm{I}^{\circ} \text {, } \\
\text { incomplete right bundle branch } \\
\text { block }\end{array}$ & c. $2680 \mathrm{C}>\mathrm{T}\left(\mathrm{p} . \operatorname{Arg} 894^{*}\right)$ & c.696G >A (p.Glu232Glu) \\
\hline 41 & $\mathbf{F}$ & 54 & CLCN1 & $\mathbf{5 0}$ & $\begin{array}{l}\text { AV-block } \text { III }^{\circ} \text {, pacemaker } \\
\text { implantation }\end{array}$ & c. $313 \mathrm{C}>\mathrm{T}$ (p.Arg105Cys) & c.501C > G (p.Phe167Leu) \\
\hline 71 & $\mathbf{F}$ & 40 & CLCN1 & 30 & $\begin{array}{l}\text { AV-block } \mathrm{II}^{\circ} \text { and syncope, pace- } \\
\text { maker implantation }\end{array}$ & c. $1649 \mathrm{C}>\mathrm{G}$ (p.Thr550Arg) & c. $1649 \mathrm{C}>\mathrm{G}$ (p.Thr550Arg) \\
\hline
\end{tabular}

Bold $=$ cases of interest

$A V$-Block atrioventricular block

\section{Diagnostic assessments}

The family history was positive in $56 \%$ of patients $(50 \%$ NDM-CLCN1, 70\% NDM-SCN4A patients). The mean diagnostic delay was $16.6 \pm 12.2$ years, without significant difference between both types or between patients with or without positive family history for myotonia. Furthermore, there was no significant difference in diagnostic delay between patients who were genetically tested after the year $2010(16 \pm 11.9$ years $)$ and those before $(19.1 \pm 14.2$ years $)$ $(p=0.55)$.

$40.5 \%$ of NDM-CLCN 1 and $38.1 \%$ of NDM-SCN4A patients had normal $\mathrm{CK}$ values. In patients with elevated CK, the CK was on average 2-3 times upper normal limit with no relevant differences in both groups (median and IQR in NDM-CLCN1: 286.0 U/L, 226.5-467.7 U/L; median and IQR in NDM-SCN4A: 373.7 U/L, 213.4-1106.8 U/L).

The time between the onset of the disease and the performance of the first EMG was on average 12.6 \pm 10.7 years, with no significant differences between NDM-CLCN1 and NDM-SCN4A $(14.2 \pm 11.4$ years for NDM-CLCN1 and $8.7 \pm 7.9$ years in NDM-SCN4A; $p=0.152$ ). EMG was normal in only $7.7 \%$ of the patients. Myotonic runs were detected in $86.2 \%$ of patients in their first documented EMG, other forms of pathological spontaneous activity as fibrillation potentials and pseudo-myotonic runs were present in $18.5 \%$ of the patients.

A genetic confirmation was obtained in all patients. In 24/70 no additional genes were tested before reaching the correct diagnosis (Supplementary Table 1). In the remaining 46/70 patients, a tandem analysis of $S C N 4 A$ and $C L C N 1$ was performed in $15.2 \%$ and next generation sequencing techniques were adopted in $58.9 \%$ of cases. In addition to other genes responsible for NDM, the most common differential diagnosis was myotonic dystrophy, investigated in $47.1 \%$ of patients.

The genotype of all patients is shown in Table 4. Half of NDM-CLCN1-myotonia patients $(n=24)$ had at least one heterozygous c.2680C $>\mathrm{T}$ mutation. The most common mutations in SCN4A were the c.3917G $>\mathrm{T}(n=5)$, c. $1333 \mathrm{G}>\mathrm{A}(n=4)$ and $\mathrm{c} .2111 \mathrm{C}>\mathrm{T}(n=3)$. A total of 12 new genetic variants could be identified (highlighted in bold in Table 3). Newly identified mutations included frameshift, in-frame, missense, and splice-site mutations. The patients with novel variants did not show peculiar clinical features different from those of other patients with known CLCN1 or SCN4A mutations.

\section{Treatment}

The mean follow-up duration for treatment was $4.3 \pm 6$ years, with no significant differences between patients with NDMCLCN1 or NDM-SCN4A $(p=0.92)$. From the review of all patients' records, $13.3 \%$ of patients have never taken an anti-myotonic drug (11.4\% of CLCN1 and $20 \%$ of SCN4A, $p=0.6)$. At the last follow-up visit, $48.9 \%$ of patients $(44.7 \%$ of NDM-CLCN1, 63.6\% NDM-SCN4A) were not taking any anti-myotonic medication, either because the symptoms' burden was too low or because of the low efficacy or side effects of the drugs tested. All patients being treated with anti-myotonic drugs (51\%) received a monotherapy. On average, two different anti-myotonic drugs were tested in the past (range 1-5). A list of the reported medications 
Table 4 Mutational spectrum

\begin{tabular}{|c|c|c|c|c|c|c|}
\hline Pt. ID & Gene & $\begin{array}{l}\text { Inheritance in } \\
\text { the family* }\end{array}$ & Mutation 1 & Mutation 1 & Mutation 2 & Mutation 2 \\
\hline 1 & CLCN1 & $\mathrm{AR}$ & c. $407 \mathrm{~A}>\mathrm{G}$ & p. Asp136Gly & n.f & n.f \\
\hline 3 & CLCN1 & $\mathrm{AR}$ & c.1437_1450del & p.Pro480Hisfs $* 24$ & c.2422_2427dup & p.Glu808_GIn809dup \\
\hline 4 & CLCN1 & $\mathrm{AD}$ & c. $2680 \mathrm{C}>\mathrm{T}$ & p.Arg894* & c. $2680 \mathrm{C}>\mathrm{T}$ & p.Arg894* \\
\hline 5 & CLCN1 & $\mathrm{AD} / \mathrm{AR}$ & c. $937 \mathrm{G}>\mathrm{A}$ & p.Ala313Thr & c. $2680 \mathrm{C}>\mathrm{T}$ & p.Arg894* \\
\hline 6 & CLCN1 & $\mathrm{AR}$ & c.1437_1450del & p.Pro 480 Hisfs $* 24$ & c. $2680 \mathrm{C}>\mathrm{T}$ & p.Arg894* \\
\hline 8 & CLCN1 & $\mathrm{AD}$ & c. $2680 \mathrm{C}>\mathrm{T}$ & p.Arg894* & & \\
\hline 9 & CLCN1 & $\mathrm{AR}$ & c. $1444 \mathrm{G}>\mathrm{A}$ & p. Gly482Arg & n.f & n.f \\
\hline 10 & CLCN1 & $\mathrm{AD}$ & c. $870 \mathrm{C}>\mathrm{G}$ & p.Ile290Met & & \\
\hline 12 & CLCN1 & $\mathrm{AR}$ & c. $2680 \mathrm{C}>\mathrm{T}$ & p.Arg894* & c. $180+3 \mathrm{~A}>\mathrm{T}$ & p.Gln60ins*22 \\
\hline 13 & CLCN1 & $\mathrm{AR}$ & c. $2680 \mathrm{C}>\mathrm{T}$ & p.Arg894* & c. $2680 \mathrm{C}>\mathrm{T}$ & p.Arg894* \\
\hline 20 & CLCN1 & $\mathrm{AR}$ & c. $1013 \mathrm{G}>\mathrm{A}$ & p.Arg338Gln & c. $1478 \mathrm{C}>\mathrm{A}$ & p.Ala493Glu \\
\hline 22 & CLCN1 & $\mathrm{AR}$ & c. $180+3 \mathrm{~A}>\mathrm{T}$ & p.Gln60ins*22 & c. $180+3 \mathrm{~A}>\mathrm{T}$ & p.Gln60ins*22 \\
\hline 27 & CLCN1 & $\mathrm{AD}$ & c. $2680 \mathrm{C}>\mathrm{T}$ & p.Arg894* & c. $2564 \mathrm{G}>\mathrm{A}$ & p.Gly855Glu \\
\hline 29 & CLCN1 & $\mathrm{AR}$ & c. $2680 \mathrm{C}>\mathrm{T}$ & p.Arg894* & c. $1238 \mathrm{~T}>\mathrm{G}$ & p.Phe413Cys \\
\hline 30 & CLCN1 & $\mathrm{AD}$ & c. $2680 \mathrm{C}>\mathrm{T}$ & p.Arg894* & & \\
\hline 31 & CLCN1 & $\mathrm{AD}$ & c. $937 \mathrm{G}>\mathrm{A}$ & p.Ala313Thr & & \\
\hline 32 & CLCN1 & $\mathrm{AR}$ & c. $2680 \mathrm{C}>\mathrm{T}$ & p.Arg894* & c.696G $>A$ & p.Glu232Glu \\
\hline 33 & CLCN1 & $\mathrm{AD}$ & c. $2680 \mathrm{C}>\mathrm{T}$ & p.Arg894* & & \\
\hline 34 & CLCN1 & $\mathrm{AD}$ & c.568G $>A$ & p.Gly190Arg & & \\
\hline 35 & CLCN1 & $\mathrm{AR}$ & c. $180+3 \mathrm{~A}>\mathrm{T}$ & p.Gln60ins*22 & c. $2680 \mathrm{C}>\mathrm{T}$ & p.Arg894* \\
\hline 36 & CLCN1 & $\mathrm{AR}$ & c. $2680 \mathrm{C}>\mathrm{T}$ & p.Arg894* & c. $2680 \mathrm{C}>\mathrm{T}$ & p.Arg894* \\
\hline 39 & CLCN1 & $\mathrm{AR}$ & c. $501 \mathrm{C}>\mathrm{G}$ & p.Phe167Leu & c.1437_1450del & p.Ile479Ilefs*25 \\
\hline 41 & CLCN1 & $\mathrm{AR}$ & c. $313 \mathrm{C}>\mathrm{T}$ & p.Arg105Cys & c. $501 \mathrm{C}>\mathrm{G}$ & p.Phe167Leu \\
\hline 42 & CLCN1 & $\mathrm{AD}$ & c. $983 \mathrm{C}>\mathrm{T}$ & p.Thr328Ile & & \\
\hline 43 & CLCN1 & $\mathrm{AR}$ & c. $2401 \mathrm{G}>\mathrm{T}$ & p.Glu801* & c.613G $>$ A & p.Glu205Lys \\
\hline 45 & CLCN1 & $\mathrm{AR}$ & c.2114_2115het_insT & p.Pro705ProfsX8 & & \\
\hline 46 & CLCN1 & $\mathrm{AR}$ & c. $2926 \mathrm{C}>\mathrm{T}$ & p.Arg976X & c. $1655 \mathrm{~A}>\mathrm{G}$ & p.Gln552Arg \\
\hline 47 & CLCN1 & $\mathrm{AR}$ & c. $2680 \mathrm{C}>\mathrm{T}$ & p.Arg894* & c.1444_1449delGGAGGC & p.Gly482_Gly483del \\
\hline 48 & CLCN1 & $\mathrm{AD}$ & c. $2680 \mathrm{C}>\mathrm{T}$ & p.Arg894* & & \\
\hline 49 & CLCN1 & $\mathrm{AD}$ & c. $2680 \mathrm{C}>\mathrm{T}$ & p.Arg894* & & \\
\hline 52 & CLCN1 & $\mathrm{AD}$ & c.1445G $>$ A & p.Gly482Glu & & \\
\hline 53 & CLCN1 & $\mathrm{AR}$ & c. $2680 \mathrm{C}>\mathrm{T}$ & p.Arg894* & c. $2680 \mathrm{C}>\mathrm{T}$ & p.Arg894* \\
\hline 54 & CLCN1 & $\mathrm{AD}$ & c. $937 \mathrm{G}>\mathrm{A}$ & p.Ala313Thr & & \\
\hline 55 & CLCN1 & $\mathrm{AR}$ & c. $180+3 \mathrm{~A}>\mathrm{T}$ & p.Gln60ins*22 & c. $2680 \mathrm{C}>\mathrm{T}$ & p.Arg894* \\
\hline 56 & CLCN1 & AR & c. $180+3 \mathrm{~A}>\mathrm{T}$ & p.Gln60ins $* 22$ & c. $1488 \mathrm{G}>\mathrm{T}$ & p.Arg496Ser \\
\hline 57 & CLCN1 & AR & c. $568 \mathrm{G}>\mathrm{A}$ & p.Gly190Arg & c. $1166+5 G>A$ & p.? \\
\hline 58 & CLCN1 & $\mathrm{AR}$ & c. $1238 \mathrm{~T}>\mathrm{G}$ & p.Phe413Cys & n.f & n.f \\
\hline 60 & CLCN1 & $\mathrm{AD}$ & c. $1655 \mathrm{~A}>\mathrm{G}$ & p.Gln552Arg & & \\
\hline 62 & CLCN1 & $\mathrm{AD}$ & c. $2680 \mathrm{C}>\mathrm{T}$ & p.Arg894* & & \\
\hline 63 & CLCN1 & $\mathrm{AR}$ & c. $2680 \mathrm{C}>\mathrm{T}$ & p.Arg894* & c. $1013 \mathrm{G}>\mathrm{A}$ & p.Arg338Gln \\
\hline 64 & CLCN1 & $\mathrm{AD}$ & c. $2680 \mathrm{C}>\mathrm{T}$ & p.Arg894* & & \\
\hline 65 & CLCN1 & $\mathrm{AR}$ & c. $979 \mathrm{G}>\mathrm{A}$ & p.Val327Ile & c.1437_1450del & p.Pro480Hisfs*24 \\
\hline 66 & CLCN1 & $\mathrm{AR}$ & c. $2680 \mathrm{C}>\mathrm{T}$ & p.Arg894* & c. $2680 \mathrm{C}>\mathrm{T}$ & p.Arg894* \\
\hline 67 & CLCN1 & $\mathrm{AD}$ & c. $929 \mathrm{C}>\mathrm{T}$ & p.Thr310Met & & \\
\hline 70 & CLCN1 & $\mathrm{AD}$ & c. $2680 \mathrm{C}>\mathrm{T}$ & p.Arg894* & & \\
\hline 71 & CLCN1 & $\mathrm{AR}$ & c. $1649 \mathrm{C}>\mathrm{G}$ & p.Thr550Arg & c. $1649 \mathrm{C}>\mathrm{G}$ & p.Thr550Arg \\
\hline 72 & CLCN1 & $\mathrm{AR}$ & c. $180+3 \mathrm{~A}>\mathrm{T}$ & p.Gln60ins*22 & c. $1488 \mathrm{G}>\mathrm{T}$ & p.Arg496Ser \\
\hline 73 & CLCN1 & $\mathrm{AR}$ & c. $2680 \mathrm{C}>\mathrm{T}$ & p.Arg894* & c. $2680 \mathrm{C}>\mathrm{T}$ & p.Arg894* \\
\hline 2 & SCN4A & $\mathrm{AD}$ & c. $2024 \mathrm{G}>\mathrm{A}$ & p. Arg675Gln & & \\
\hline
\end{tabular}


Table 4 (continued)

\begin{tabular}{|c|c|c|c|c|c|c|}
\hline Pt. ID & Gene & $\begin{array}{l}\text { Inheritance in } \\
\text { the family* }\end{array}$ & Mutation 1 & Mutation 1 & Mutation 2 & Mutation 2 \\
\hline 7 & SCN4A & $\mathrm{AD}$ & c. $2111 \mathrm{C}>\mathrm{T}$ & p.Thr704Met & & \\
\hline 11 & SCN4A & $\mathrm{AD}$ & c. $4106 \mathrm{C}>\mathrm{A}$ & p.Thr1369Asn & & \\
\hline 14 & SCN4A & $\mathrm{AD}$ & c. $3917 \mathrm{G}>\mathrm{C}$ & p.Gly1306Ala & & \\
\hline 15 & SCN4A & $\mathrm{AD}$ & c. $1333 G>T$ & p.Val445Leu & & \\
\hline 16 & SCN4A & $\mathrm{AD}$ & c. $2078 \mathrm{~T}>\mathrm{C}$ & p.Ile693Thr & & \\
\hline 17 & SCN4A & $\mathrm{AD}$ & c. $1333 \mathrm{G}>\mathrm{A}$ & p.Val445Met & & \\
\hline 18 & SCN4A & $\mathrm{AD}$ & c. $1333 \mathrm{G}>\mathrm{A}$ & p.Val445Met & & \\
\hline 19 & SCN4A & $\mathrm{AD}$ & c. $1333 \mathrm{G}>\mathrm{A}$ & p.Val445Met & & \\
\hline 21 & SCN4A & $\mathrm{AD}$ & c. $3917 \mathrm{G}>\mathrm{T}$ & p.Gly1306Val & c. $3917 \mathrm{G}>\mathrm{T}$ & p.Gly1306Val \\
\hline 23 & SCN4A & $\mathrm{AD}$ & c. $5113 \mathrm{~T}>\mathrm{A}$ & p.Phe1705Ile & & \\
\hline 24 & SCN4A & $\mathrm{AD}$ & c. $2111 \mathrm{C}>\mathrm{T}$ & p.Thr704Met & & \\
\hline 26 & SCN4A & $\mathrm{AD}$ & c. $3917 \mathrm{G}>\mathrm{C}$ & p.Gly1306Ala & & \\
\hline 28 & SCN4A & $\mathrm{AD}$ & c. $3877 \mathrm{G}>\mathrm{A}$ & p.Val1293Ile & & \\
\hline 37 & SCN4A & $\mathrm{AD}$ & c. $4342 \mathrm{C}>\mathrm{T}$ & p.Arg1448Cys & & \\
\hline 40 & SCN4A & $\mathrm{AD}$ & c. $1333 \mathrm{G}>\mathrm{A}$ & p.Val445Met & & \\
\hline 51 & SCN4A & $\mathrm{AD}$ & c. $3893 \mathrm{~T}>\mathrm{G}$ & p.Phe1298Cys & & \\
\hline 61 & SCN4A & $\mathrm{AD}$ & c. $3917 \mathrm{G}>\mathrm{T}$ & p.Gly1306Val & & \\
\hline 69 & SCN4A & $\mathrm{AD}$ & c. $2111 \mathrm{C}>\mathrm{T}$ & p.Thr704Met & & \\
\hline 74 & SCN4A & $\mathrm{AD}$ & c. $3917 \mathrm{G}>\mathrm{T}$ & p.Gly1306Val & & \\
\hline 75 & SCN4A & $\mathrm{AD}$ & c. $3917 \mathrm{G}>\mathrm{T}$ & p.Gly1306Val & & \\
\hline 76 & SCN4A & $\mathrm{AD}$ & c. $3917 \mathrm{G}>\mathrm{T}$ & p.Gly1306Val & & \\
\hline
\end{tabular}

*Some mutations in the CLCN1 gene have both a dominant or a recessive effect (e.g., p.R894*, p.A313T)

is provided in Table 5, including the level of patients' perceived efficacy, documented by the treating physician.

\section{Discussion}

In this study, we depicted the clinical features of a large cohort of German patients with NDM thus adding valuable information to the few reported large cohorts of patients (Table 1) [12, 18, 21-27]. The core clinical features of our NDM cohort were mostly confirmatory of literature data, highlighting the significant clinical overlap between patients with NDM-CLCN1 and NDM-SCN4A. However, some new findings could be summarized. The age of onset could be collocated in the 1st to 2 nd decade of life in most patients. Myotonia was the most prominent symptom, occurring more frequently in facial muscles in NDM-SCN4A and not significantly but tendentially more frequently in the legs for NDM-CLCN1, muscle weakness tended to occur more frequently in NDM-CLCN1. Myalgia was, immediately after myotonia, the most prevalent symptom during disease progression involving $52-57 \%$ of patients, without significant differences between NDM-CLCN1 and NDM-SCN4A. In the literature, the prevalence of pain in NDMs widely ranges from 28 to $53 \%$ in NDM-CLCN1 and from 56 to
$82 \%$ in NDM-SCN4A [12, 23, 24]. Some SCN4A mutations are known to be associated with myalgia (e.g., c.1333G $>$ A, c. $3917 \mathrm{G}>\mathrm{C}, \mathrm{c} .3466 \mathrm{G}>\mathrm{A}$ ), in our patients only $6 / 22$ showed either c.1333G $>$ A or the c.3917G $>$ C mutation, thus explaining only a minority of myalgic patients. The presence of pain is one of the major determinants of low quality of life in NDM patients [28] and needs to be properly monitored and treated. Some myotonia characteristics are considered more archetypal for one form or the other, as warm-up phenomenon and muscle hypertrophy usually points toward NDMCLCN1 and cold sensitivity toward NDM-SCN4A. In contrast, in our genetically confirmed cohort a more relevant clinical overlap was observed as the muscle hypertrophy, the warm-up phenomenon as well as grip- and percussion myotonia were frequently observed in both groups. Even the classic triggers were present in both NDM groups with an even higher prevalence of cold sensitivity for NDM-CLCN1 patients. This finding is untypical as usually NDM-SCN4A patients refer a more prominent worsening of symptoms with cold temperatures, but it is well known that cold may worsen symptoms also in NDM-CLCN1 patients. Similar observations were also reported by Trivedi et al. [12] and should favor a genetic tandem analysis approach rather than a single gene sequencing. Extra-muscular manifestations are thought to be non-typical for NDM patients, even though the 
Table 5 Anti-myotonic treatment in NDMs

\begin{tabular}{|c|c|c|c|c|c|c|c|}
\hline \multirow[t]{2}{*}{ Drug } & \multirow[t]{2}{*}{ Dose (mg) } & \multirow[t]{2}{*}{$N$ (total) } & \multirow[t]{2}{*}{ NDM-CLCN1\% } & \multirow[t]{2}{*}{ NDM-SCN4A\% } & \multicolumn{3}{|c|}{$\begin{array}{l}\text { Patients' perceived efficacy (mean value } \\
\text { score) }\end{array}$} \\
\hline & & & & & $n$ & NDM-CLCN1 & NDM-SCN4A \\
\hline \multicolumn{8}{|c|}{ Commonly used anti-myotonic drugs } \\
\hline Carbamazepine & $100-1600$ & 19 & 78.9 & 21.1 & 12 & 2.9 & 3 \\
\hline Flecainide & $50-300$ & 16 & 87.5 & 12.5 & 14 & 2.5 & 3 \\
\hline Mexiletine $^{\mathrm{a}}$ & $200-600^{\mathrm{b}}$ & 15 & 80 & 20 & 10 & 1.89 & 2 \\
\hline Lamotrigine $^{\mathrm{a}}$ & $50-300$ & 8 & 75 & 25 & 6 & 2.25 & 2 \\
\hline Acetazolamide & $125-500$ & 6 & 33.3 & 66.7 & 3 & n.a & 2.3 \\
\hline Phenytoin & $100-300$ & 5 & 80 & 20 & 3 & 3 & 3 \\
\hline Mexiletine ret. $^{\mathrm{a}}$ & $360-720$ & 4 & 100 & 0 & 4 & 1.3 & 1 \\
\hline Propafenone & $150-450$ & 2 & 100 & 0 & 2 & 3 & n.a \\
\hline \multicolumn{8}{|c|}{ Other drugs with potential anti-myotonic effect } \\
\hline Tolperisone & $50-450$ & 18 & 83.3 & 16.7 & 13 & 2.9 & 3 \\
\hline Gabapentin & $100-3600$ & 11 & 72.7 & 27.3 & 8 & 2.8 & 2.7 \\
\hline Methocarbamol & $750-4500$ & 9 & 77.8 & 22.2 & 8 & 2.8 & 2.5 \\
\hline Pregabalin & $150-600$ & 5 & 80 & 20 & n.a & n.a & n.a \\
\hline Baclofen & $10-75$ & 2 & 100 & 0 & n.a & n.a & n.a \\
\hline Cannabinoid/THC & $20.58-41.16 / 2.2-6.02$ & 2 & 100 & 0 & 2 & 2 & n.a \\
\hline Oxcarbazepine & $35-150$ & 1 & 100 & 0 & n.a & n.a & n.a \\
\hline
\end{tabular}

${ }^{a}$ Anti-myotonic drugs with perceived best efficacy; $1=$ excellent, $2=$ good, $3=$ poor

${ }^{\mathrm{b}}$ One patient received up to $1200 \mathrm{mg}$ with major side effects

role of SCN4A variants in causing cardiac arrhythmias and Brugada syndrome remains debated [29]. In our cohort, no patient with $S C N 4 A$ mutations presented relevant cardiac involvement; on the other hand, six NDM-CLCN1 patients had cardiac arrhythmias or conduction defects, requiring the implantation of a pacemaker in three patients. In particular, patient 65 required pacemaker implantation at age 30 years and has a positive family history of dilative cardiomyopathy documented in her father's and brother's medical history, the latter also presented with a NDM-CLCN1-myotonia. Given the autosomal recessive nature of the two known pathogenic CLCN1 gene mutations in this patient (c. $180+3 \mathrm{~A}>\mathrm{T}$ and c. $1488 \mathrm{G}>\mathrm{T}$ ), we interpret this case as an obvious doubletrouble situation. The $\mathrm{ClC}-1$ channel is mainly expressed in the skeletal muscle and only at limited levels in smooth muscle, kidney, heart, liver, and CNS [30, 31]. Since no extramuscular and especially cardiac manifestations of NDM are reported in other NDM-CLCN1 cohorts so far and given the low number of patients with cardiac arrhythmias in our cohort, a causal relation between $C L C N 1$ mutation and cardiac manifestations stays unlikely. However, NDM patients must undergo regular cardiac evaluation as the intake of anti-myotonic drugs can unmask latent and potentially lifethreatening arrhythmias.

The mean diagnostic delay of this cohort was quite long $(16.6 \pm 12.2$ years $)$, even for a rare disease. Similar results were however reported by Baumann et al. (18 \pm 14 years) and Trip et al. (12.0 \pm 10.4 years) [21, 24]. From our results, it appears that the longest delay occurred between the onset of symptoms and first EMG performed (12.6 \pm 10.7 years). This suggests that patients are often not immediately referred to a neurologist, being misdiagnosed as fibromyalgia or chronic pain syndrome; in other cases, patients with mildly pronounced symptoms, negative family history, normal CK will not seek immediate medical consultation. A normal CK was found in $39.6 \%$ of our patients, thus this should not prevent the suspicion of NDM or the referral to a neurologist. In the current German guidelines on NDM, the measurement of $\mathrm{CK}$ is a mandatory part of the diagnostic testing and is stated to be no more than $5 \times$ upper normal limits (UNL) for NDMCLCN1 and often more than twice the UNL for SCN4Amyotonias [11]. In some patients with SCN4A-myotonia, we observed CK elevation up to $1100 \mathrm{U} / \mathrm{L}$. These values may have been influenced by various factors, such as physical activity on the previous day. The EMG detected myotonic runs in $89 \%$ of NDM-CLCN1 and $80 \%$ of NDM-SCN4A patients without provocative cold test and confirmed to be the most valuable diagnostic tool for the identification of NDM patients and indication of genetic testing.

In our NDM-CLCN1 cohort, the common mutation c. $2680 \mathrm{C}>\mathrm{T}$ (p.Arg894*) was found in 50\%. This typical Thomsen CLCN1 mutation, confirmed in the original Thomsen family from Northern Germany, is also frequently found in Northern Scandinavia, Russia, Denmark and to a lesser 
extent in The Netherlands, Spain, and Italy [32, 33]. It still has a high prevalence in northern Europe. Other mutations were most frequent in Spain $(\mathrm{c} .180+3 \mathrm{~A}>\mathrm{T})$, the Netherlands (c.1238T > G, p.F413C), and Italy (c.501C > G, p.F167L) [25, 34-36]. In our study, no clear genotype-phenotype correlations could be identified, this might be also related to the several different mutations that our patients presented throughout the genes. Some previous functional studies have demonstrated how the location of the mutations in the different channel domains deeply influences the clinical severity and response to therapy producing major differences among patients sharing the same gene defect but different mutations. On the other hand, different phenotypes have also been described in patients with the same mutation, suggesting that modifier genes and environmental condition might influence the clinical features of individual patients [37, 38].

We found 12 novel genetic variants. Although we did not perform functional studies, we included some class 3 mutations that were highly suggestive of being pathogenic due to investigation of family members, indicative clinical symptoms and the evidence of EMG myotonia. Very interestingly, patient 21 displayed the dominant c. $3917 \mathrm{G}>\mathrm{T}$ mutation on both alleles. This change from glycine to valine is known to cause moderate to severe myotonia [39] and this patient displayed earlier and more severe symptoms compared to other affected family members.

The main differential diagnosis of NDM were dystrophic myotonias, with a national biased approach, like myotonic dystrophy type 2 being tested slightly more frequently than myotonic dystrophy type 1 in our sample. Other genes examined in these NDM patients were CAV3, ATP2Al (Brody disease) and $H I N T 1$ (hereditary neuromyotonia with axonal neuropathy). Since 2010 next-generation sequencing can be used in the clinical setting in Germany. Therefore, we wanted to examine whether this new sequencing technique reduced the diagnostic delay of NDMs. However, no significant differences were observed for the diagnostic delay in patients diagnosed before and after 2010. Probably because even before 2010 tandem analysis of CLCN1 and SCN4A was commonly conducted.

The effective symptomatic treatment of NDM remains a challenge. At their last follow-up visit, about $50 \%$ of our patients (44.7\% NDM-CLCN1, 63.6\% NDM-SCN4A) were not taking any anti-myotonic therapy. Trivedi et al. reported the use of anti-myotonic medication in $60.6 \%$ of patients, Dupré et al. in $41 \%$ of recessive (36\% with significant improvement), $0 \%$ of dominant NDM-CLCN1 patients and $43 \%$ of NDM-SCN4A patients. As described by Dupré et al., phenytoin and gabapentin were most effective in patients with recessive CLCN1-myotonia, while mexiletine and carbamazepine were most effective in patients with SCN4A-myotonia [12, 22]. The most frequently used anti-myotonic drugs were carbamazepine, flecainide, mexiletine and lamotrigine in this order of frequency. Flecainide and mexiletine are the drugs of first choice according to the current German guidelines, carbamazepine was more used in the past and lamotrigine more recently after the clinical study by Anderson et al. [11, 16] Patients rated mexiletine, and lamotrigine as the most effective therapies, in accordance with recent RCTs [15, 16], whereas other medications were mainly considered as not satisfactory. This is also confirmed by the high number of tested anti-myotonic drugs (up to 11 different drugs). The good efficacy of phenytoin/gabapentin in recessive CLCN1-myotonia and carbamazepine and mexiletine in SCN4A-myotonia reported by Dupré et al. was, with exception of mexiletine, not observed in our cohort [22]. Our data however, lack information regarding the duration of anti-myotonic drug intake and patients' compliance, which may impact the evaluation of drug efficacy.

The limitation of our study is its retrospective design. The collection of information has been likely affected by the accuracy of different physicians in record keeping. Despite this, the strength of our study lies in a large number of genetically confirmed patients, and the opportunity of a direct comparison of patients with either a $C L C N 1$ or a $S C N 4 A$ gene mutation in one single highly specialized neuromuscular center over 25 years.

In summary, this study highlights the clinical, genetic and therapeutic challenges related to the diagnosis and management of NDM patients and unmet needs are the lack of follow-up studies of large cohorts with reliable assessments of disease progression and treatment efficacy.

Author contributions NV contributed to data collection, analysis and drafting of the manuscript. FM contributed to study design, data analysis and drafting and review of the manuscript, DG contributed to revision and interpretation of the genetic data and review of the manuscript. BS contributed with conception of the study, study design, data analysis and review of the manuscript. This manuscript is part of the medical thesis of NV.

Funding Open Access funding enabled and organized by Projekt DEAL.

Code availability Not applicable.

\section{Compliance with ethical standards}

Conflicts of interest N. V. and D. G. declare that they have no conflict of interest. F. M. has participated to one advisory board meeting of Lupin therapeutics, B. S. is a scientific advisor of Lupin therapeutics.

Ethics standards The study has been conducted in compliance with the principles of the declaration of Helsinki and with the local German laws and regulations.

Consent to participate Not applicable. 
Consent for publication All authors agreed to the publication of the manuscript in its current form.

Availability of data and material Available upon reasonable request to the corresponding author.

Open Access This article is licensed under a Creative Commons Attribution 4.0 International License, which permits use, sharing, adaptation, distribution and reproduction in any medium or format, as long as you give appropriate credit to the original author(s) and the source, provide a link to the Creative Commons licence, and indicate if changes were made. The images or other third party material in this article are included in the article's Creative Commons licence, unless indicated otherwise in a credit line to the material. If material is not included in the article's Creative Commons licence and your intended use is not permitted by statutory regulation or exceeds the permitted use, you will need to obtain permission directly from the copyright holder. To view a copy of this licence, visit http://creativecommons.org/licenses/by/4.0/.

\section{References}

1. Hudson A, Ebers G, Bulman D (1995) The skeletal muscle sodium and chloride channel diseases. Brain 118(2):547-563

2. Jurkat-Rott K, Lerche H, Lehmann-Horn F (2011) Muskuläre Kanalopathien. Der Nervenarzt 82(4):511-521

3. Morales F, Pusch M (2020) An up-to-date overview of the complexity of genotype-phenotype relationships in myotonic channelopathies. Front Neurol 10:1404

4. Jurkat-Rott K (2005) Muscle channelopathies and critical points in functional and genetic studies. J Clin Investig 115(8):2000-2009

5. Matthews E et al (2010) The non-dystrophic myotonias: molecular pathogenesis, diagnosis and treatment. Brain 133(1):9-22

6. Lipicky RJ, Bryant SH, Salmon JH (1971) Cable parameters, sodium, potassium, chloride, and water content, and potassium efflux in isolated external intercostal muscle of normal volunteers and patients with myotonia congenita. J Clin Investig 50(10):2091-2103

7. Yang N et al (1994) Sodium channel mutations in paramyotonia congenita exhibit similar biophysical phenotypes in vitro. Proc Natl Acad Sci 91(26):12785-12789

8. Montagnese F, Schoser B (2018) Dystrophische und nicht-dystrophische Myotonien. Fortschritte der Neurologie Psychiatrie 86(09):575-583

9. Heatwole CR, Moxley RT (2007) The nondystrophic myotonias. Neurotherapeutics 4(2):238-251

10. Stunnenberg B et al (2020) Guidelines on clinical presentation and management of non-dystrophic myotonias. Muscle Nerve 62(4):430-444

11. Schneider-Gold C et al (2018) Myotone Dystrophien, nicht dystrophe Myotonien und periodische Paralysen. Aktuelle Neurologie 45(03): 167-177

12. Trivedi JR et al (2013) Non-dystrophic myotonia: prospective study of objective and patient reported outcomes. Brain 136(7):2189-2200

13. Fournier E et al (2004) Electromyography guides toward subgroups of mutations in muscle channelopathies. Ann Neurol 56(5):650-661

14. Statland JM (2012) Mexiletine for symptoms and signs of myotonia in nondystrophic myotonia. JAMA 308(13): 1357

15. Stunnenberg BC et al (2018) Effect of mexiletine on muscle stiffness in patients with nondystrophic myotonia evaluated using aggregated N-of-1 trials. JAMA 320(22):2344
16. Andersen $\mathrm{G}$ et al (2017) The antimyotonic effect of lamotrigine in non-dystrophic myotonias: a double-blind randomized study. Brain 140(9):2295-2305

17. Montagnese $\mathrm{F}$ et al (2020) A role for cannabinoids in the treatment of myotonia? Report of compassionate use in a small cohort of patients. J Neurol 267(2):415-421

18. Koch MC et al (1993) Evidence for genetic homogeneity in autosomal recessive generalised myotonia (Becker). J Med Genet 30(11):914-917

19. HIV/AIDS, definition of key terms, consolidated ARV guidelines. 2013 09.04.2020]. https://www.who.int/hiv/pub/guidelines/arv20 13/intro/keyterms/en/. Accessed 09 Apr 2020

20. Petry NM (2002) A comparison of young, middle-aged, and older adult treatment-seeking pathological gamblers. Gerontologist 42(1):92-99

21. Baumann P, Myllyla VV, Leisti J (1998) Myotonia congenita in northern Finland: an epidemiological and genetic study. J Med Genet 35(4):293-296

22. Dupré $\mathrm{N}$ et al (2009) Clinical, electrophysiologic, and genetic study of non-dystrophic myotonia in French-Canadians. Neuromuscul Disord 19(5):330-334

23. Statland JM et al (2011) An interactive voice response diary for patients with non-dystrophic myotonia. Muscle Nerve 44(1):30-35

24. Trip J et al (2009) Redefining the clinical phenotypes of nondystrophic myotonic syndromes. J Neurol Neurosurg Psychiatry 80(6):647-652

25. Trip J et al (2008) In tandem analysis of CLCN1 and SCN4A greatly enhances mutation detection in families with non-dystrophic myotonia. Eur J Hum Genet 16(8):921-929

26. Sansone VA et al (2012) Measuring quality of life impairment in skeletal muscle channelopathies. Eur J Neurol 19(11):1470-1476

27. Becker PE et al (1977) Myotonia congenita and syndromes associated with myotonia. Topics in human genetics, vol 3. Thieme, Stuttgart

28. Trip J et al (2009) Health status in non-dystrophic myotonias: close relation with pain and fatigue. J Neurol 256(6):939-947

29. Bissay V et al (2016) SCN4A variants and Brugada syndrome: phenotypic and genotypic overlap between cardiac and skeletal muscle sodium channelopathies. Eur J Hum Genet 24(3):400-407

30. Steinmeyer K, Ortland C, Jentsch TJ (1991) Primary structure and functional expression of a developmentally regulated skeletal muscle chloride channel. Nature 354(6351):301-304

31. Chen TT et al (2013) Novel brain expression of ClC-1 chloride channels and enrichment of CLCN1 variants in epilepsy. Neurology 80(12):1078-1085

32. Skálová D et al (2013) CLCN1 mutations in Czech patients with myotonia congenita, in silico analysis of novel and known mutations in the human dimeric skeletal muscle chloride channel. PLoS ONE 8(12):e82549

33. Sun C et al (2001) Spectrum of CLCN1 mutations in patients with myotonia congenita in Northern Scandinavia. Eur J Hum Genet 9(12):903-909

34. Mazón MJ et al (2012) Screening for mutations in Spanish families with myotonia. Functional analysis of novel mutations in CLCN1 gene. Neuromusc Disord 22(3):231-243

35. Brugnoni R et al (2013) A large cohort of myotonia congenita probands: novel mutations and a high-frequency mutation region in exons 4 and 5 of the CLCN1 gene. J Hum Genet 58(9):581-587

36. Modoni A et al (2011) Low-rate repetitive nerve stimulation protocol in an Italian cohort of patients affected by recessive myotonia congenita. J Clin Neurophysiol 28(1):39-44

37. Imbrici P et al (2015) ClC-1 mutations in myotonia congenita patients: insights into molecular gating mechanisms and genotypephenotype correlation. J Physiol 593(18):4181-4199 
38. Farinato A et al (2019) Pharmacogenetics of myotonic hNav1.4 sodium channel variants situated near the fast inactivation gate. Pharmacol Res 141:224-235
39. Mitrovié N et al (1995) Different effects on gating of three myotonia-causing mutations in the inactivation gate of the human muscle sodium channel. J Physiol 487(1):107-114 